\title{
$R R 1$ and $R R 2$ gene deletion affects the immunogenicity of a live attenuated pseudorabies virus vaccine candidate in natural pig host
}

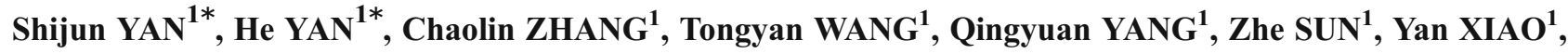 \\ Feifei TAN ${ }^{1}$, Xiangdong LI $(\bowtie)^{1}$, Kegong TIAN $(\bowtie)^{1,2}$ \\ 1 National Research Center for Veterinary Medicine, Luoyang 471003, China \\ 2 College of Animal Science and Veterinary Medicine, Henan Agricultural University, Zhengzhou 450002, China
}

\begin{abstract}
As virulence-determining genes, $R R 1$ and $R R 2$ encode the small subunit and large subunit of viral ribonucleotide reductase (RR) in pseudorabies virus which have been extensively studied in mice. However, their role in pigs has not been adequately investigated. In this study, we deleted $R R 1$ and $R R 2$ genes based on a $T K / g E / g I$ triple gene-deleted pseudorabies virus and tested its efficacy in pigs as a vaccine candidate. The rescued virus showed similar growth properties and plaque size in vitro as its parent strain. In an animal study, the virus could elicit humoral immune responses shown by generation of gB-specific antibodies and virus neutralizing antibodies. However, vaccination could not provide protection against virulent pseudorabies virus challenge since vaccinated pigs showed clinical pseudorabies-specific syndromes. The deficiency in protection may due to the generation of late and low levels of $\mathrm{gB}$ antibodies and virus neutralizing antibodies.
\end{abstract}

Keywords pseudorabies virus, $R R 1$ and $R R 2$, ribonucleotide reductase, vaccine candidate

\section{Introduction}

Pseudorabies (PR) is an acute disease which has a wide range of hosts including ruminants, carnivores, rodents and pigs $^{[1]}$. The causative agent, pseudorabies virus (PRV), belongs to family Herpesviridae, subfamily Alphaherpesvirinae, and is placed in the genus Varicellovirus. PRV has

Received December 17, 2015; accepted February 17, 2016

Correspondences: xiaonanzhong@163.com, tiankg@263.net

${ }^{*}$ These authors contributed equally to the work been successfully eradicated in western countries by using gene-deleted modified live vaccines accompanied by the application of ELISA tests ${ }^{[2]}$. However, with the emergence of PRV variants, this disease is still prevalent in China and poses a huge threat to the swine industry ${ }^{[3]}$.

As for PRV modified live vaccines, several virulencerelated genes such as thymidine kinase (TK), and glycoproteins glycoprotein E (gE) and glycoprotein I (gI) are commonly targeted for deletion to reduce virulence but keep immunogenicity ${ }^{[4]}$. By using bacterial artificial chromosome (BAC) technology, double- or triple-gene deleted vaccines were constructed and showed to provide useful protection as vaccine candidates ${ }^{[5,6]}$. In PRV, ribonucleotide reductase (RR) genes including $R R 1$ (UL39) and $R R 2$ (UL40) encode ribonucleotide reductase that reduces ribonucleoside diphosphates to the corresponding deoxyribonucleoside diphosphates and is a determinant of virus virulence in mice ${ }^{[7]}$. However, the roles of $R R$ genes in PRV in the natural pig have seldom been reported. In this study, we successfully rescued a $R R 1 / R R 2$-deleted virus based on previously constructed $T K / g E / g I$ triple gene-deleted pseudorabies virus and tested its efficacy on pigs as a vaccine candidate.

\section{Materials and methods}

\subsection{Construction of gene-deleted virus}

The construction of $T K / g E / g I$ triple gene-deleted pseudorabies virus (vPRV) has been described previously ${ }^{[4]}$. This strategy was used to construct $R R$ gene-deleted virus based on the triple gene-deleted virus. Briefly, pBAC-PRV $\mathrm{TK}^{-} / \mathrm{gE}^{-} / \mathrm{gI}^{-}$plasmid was transformed into Escherichia coli DY380 and positive colonies were made into 
competent cells. A PCR product containing the kanamycin resistant gene with short sequence duplication of PRV $R R$ genes was then transformed into the above competent cells. After digestion with I-SceI, the linear plasmid was again transformed into E. coli DY380 to remove the kanamycin gene and to construct $\mathrm{pBAC}-\mathrm{PRV} \mathrm{TK}^{-} / \mathrm{gE}^{-} / \mathrm{gI}^{-} / \mathrm{RR}^{-}$ plasmid. To remove the BAC gene cassette, the plasmid was co-transfected with pBS 185 plasmid in Vero cells and $T K / g E / g I / R R$ gene-deleted virus ( $\mathrm{vPRV} \mathrm{TK}^{-} / \mathrm{gE}^{-} / \mathrm{gI}^{-} / \mathrm{RR}^{-}$) was obtained after purification by plaque assay. Plaque sizes were determined at $48 \mathrm{~h}$ by inoculating $1000 \mathrm{TCID}_{50}$ of virus on Vero cells. After $1 \mathrm{~h}$ incubation with virus, the medium was aspirated and cells were overlaid with $1 \%$ low-melting point agarose containing 2\% FBS in DMEM for plaque formation. For each virus, 100 plaques were randomly selected and their size determined by Image J software (National Institutes of Health). Values were calculated in comparison to those of $T K / g E / g I$ triple gene-deleted PRV which was set at $100 \%$. Average percentages and standard deviations were determined from three independent experiments ${ }^{[4]}$.

\subsection{Animals and experiment design}

Fifteen 3-week-old piglets free of PRV, porcine reproductive and respiratory syndrome virus, classical swine fever virus, and porcine circovirus 2 were randomly divided into 3 groups. In the first two groups, pigs were vaccinated

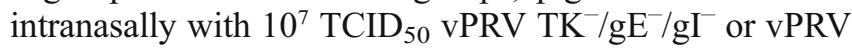
$\mathrm{TK}^{-} / \mathrm{gE}^{-} / \mathrm{gI}^{-} / \mathrm{RR}^{-}$, respectively. Pigs in the unvaccinated group (CC group) received Dulbecco minimum essential medium. After vaccination, rectal temperature and clinical signs were recorded on a daily basis. The clinical score (range from 1 to 4) was evaluated on a daily basis as previously described ${ }^{[8]}$. Four weeks after vaccination, all pigs were challenged intranasally with $1 \times 10^{7} \mathrm{TCID}_{50}$ virulent PRV variant HN1201. At 7 days post-challenge (dpc), all surviving pigs were euthanized and necropsied. The animal trial was approved by the Animal Care and Ethics Committee of China National Research Center for Veterinary Medicine.

2.3 Enzyme-linked immunosorbent assay and serum-virus neutralizing test (SNT)

The serum samples were collected to monitor PRV gBspecific antibody responses at designated days according to the manufacturer's directions (IDEXX, Westbrook, ME, USA). The cutoff $\mathrm{S} / \mathrm{N}$ values for the assay are: negative as greater than 0.70 , positive as less than or equal to 0.60 , and suspect as greater than 0.60 and less than or equal to 0.70 .

Serum samples were tested by SNT for the PRV-specific neutralizing antibodies. Briefiy, serum samples were heat inactivated at $56^{\circ} \mathrm{C}$ for $30 \mathrm{~min}$ prior to performing the serum-neutralization assay. Two fold serially diluted sera $(50 \mu \mathrm{L})$ were mixed with an equal volume $100 \mathrm{TCID}_{50}$ of the PRV HN1201 in 96-well culture plates and incubated at $37^{\circ} \mathrm{C}$ for $1 \mathrm{~h}$ in $5 \% \mathrm{CO}_{2}$ atmosphere. After incubation, $100 \mu \mathrm{L}$ of PK-15 cell suspension containing $2 \times 10^{4}$ cells was added to each well. The inoculated cells were then incubated at $37^{\circ} \mathrm{C}$ for 5 days for development of CPE to determine the titers of PRV-specific NAbs, and the titers were expressed as the reciprocal of the highest dilution at which infection of the PK-15 cells was inhibited in 50\% of the culture wells.

\subsection{Histopathology and immunohistochemistry staining}

The tonsil, lung, brain, cerebellum and trigeminal samples were collected for hematoxylin and eosin (H\&E) and immunohistochemistry staining. The H\&E staining was operated automatically by a Leica fully automatic dyeing machine according to standard procedures. The immunohistochemistry staining was performed as previously described $^{[9]}$. Photographs of the slides were taken at $400 \times$ magnification.

\subsection{Statistical analysis}

Data in this study was presented as mean $\pm \mathrm{SD}$. The differences of raw data among three groups were determined by using one-way ANOVA in GraphPad Prism 5.0 Software (San Diego, CA, USA). Differences were considered statistically significant when $P<0.05$.

\section{Results}

\subsection{Growth properties of gene-deleted virus}

Virus growth was delineated by determination of virus titer every $4 \mathrm{~h}$ post-infection. The growth features of vPRV $\mathrm{TK}^{-} / \mathrm{gE}^{-} / \mathrm{gI}^{-} / \mathrm{RR}^{-}$were virtually identical to parental vPRV $\mathrm{TK}^{-} / \mathrm{gE}^{-} \mathrm{gI}^{-}$in Vero cells as shown by Fig. 1a. The virus titers of these two viruses kept increasing and reached the maximum titers at $40 \mathrm{~h}$ post-infection. The plaque sizes of these two viruses were also determined at $48 \mathrm{~h}$. As shown by Fig. $1 b$, the plaque sizes of reconstituted virus were similar to parental virus.

\subsection{Antibody response after vaccination}

After vaccination, no clinical symptoms were observed in any group of pigs (data not shown). gB-specific IDEXX ELISA showed that all pigs in the $\mathrm{vPRV} \mathrm{TK}^{-} / \mathrm{gE}^{-} / \mathrm{gI}^{-}$ group were positive at 14 days post-caccination $(\mathrm{dpv})$ and three pigs in the $\mathrm{vPRV} \mathrm{TK}^{-} / \mathrm{gE}^{-} / \mathrm{g \Gamma}^{-} / \mathrm{RR}^{-}$group were positive (Fig. 2a). At $28 \mathrm{dpv}$, all pigs in both vaccinated groups were positive for $\mathrm{gB}$ antibodies, and $\mathrm{S} / \mathrm{N}$ value for the $\mathrm{vPRV} \mathrm{TK}^{-} / \mathrm{gE}^{-} / \mathrm{gI}^{-} / \mathrm{RR}^{-}$group was significantly higher than for the vPRV $\mathrm{TK}^{-} / \mathrm{gE}^{-} / \mathrm{gI}^{-}$group (Fig. 2b). Unvaccinated pigs did not develop gB antibodies. Consistent with 
gB ELSIA antibodies, the virus neutralizing antibody titers in the $\mathrm{vPRV} \mathrm{TK}^{-} / \mathrm{gE}^{-} / \mathrm{gI}^{-} / \mathrm{RR}^{-}$group were also significantly lower than vPRV $\mathrm{TK}^{-} / \mathrm{gE}^{-} / \mathrm{gI}^{-}$group (Fig. $2 \mathrm{c}$ ).

\subsection{Clinical manifestations after viral challenge}

After viral challenge, all pigs in the DMEM group showed typical PR-clinical symptoms such as fever (Fig. 3a), respiratory distress, excessive salvation and neurological signs. Also, all pigs in the $\mathrm{vPRV} \mathrm{TK}^{-} / \mathrm{gE}^{-} / \mathrm{gI}^{-} / \mathrm{RR}^{-}$group showed similar clinical manifestations as the pigs in the DMEM group with significantly higher clinical scores than for the $\mathrm{vPRV} \mathrm{TK}^{-} / \mathrm{gE}^{-} / \mathrm{gI}^{-}$group (Fig. 3b). In contrast, pigs in the $\mathrm{vPRV} \mathrm{TK}^{-} / \mathrm{gE}^{-} / \mathrm{gI}^{-}$group only showed a transient fever without other obvious clinical symptoms. No pigs were found to have died by $5 \mathrm{dpc}$ in the $\mathrm{vPRV} \mathrm{TK}^{-} / \mathrm{gE}^{-} / \mathrm{gI}^{-}$ $/ R^{-}$group (Fig. 4a). However, three pigs had died at $3 \mathrm{dpc}$ and two pigs died at 4 and $5 \mathrm{dpc}$ in the DMEM group. All pigs were weighed at $4 \mathrm{dpc}$. As shown in Fig. $4 \mathrm{~b}$, all pigs in the DMEM and $\mathrm{vPRV} \mathrm{TK}^{-} / \mathrm{gE}^{-} / \mathrm{gI}^{-} / \mathrm{RR}^{-}$groups lost
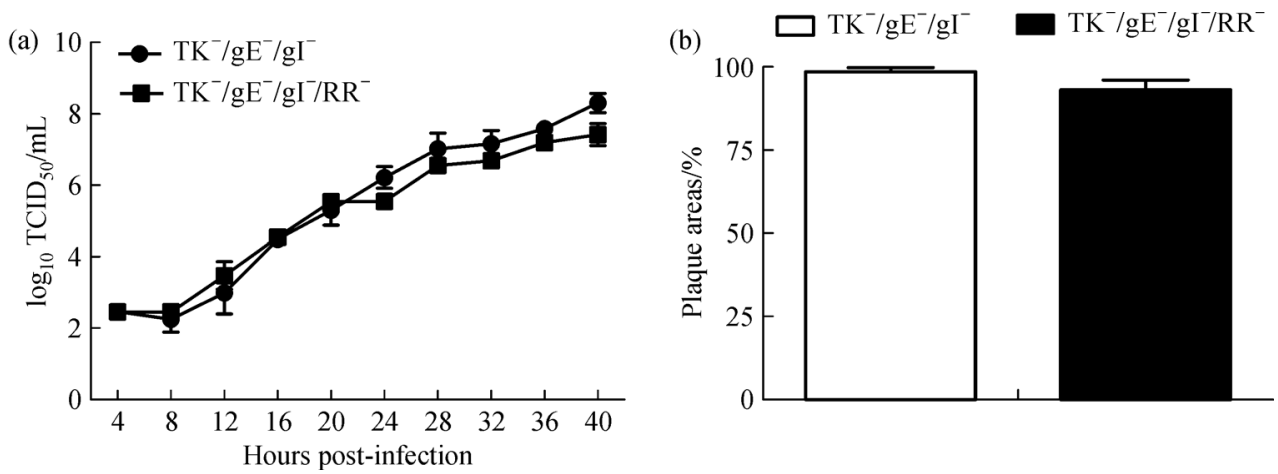

Fig. 1 Replication kinetics (a) and plaque size (b) of $\mathrm{vPRVTK}^{-} / \mathrm{gE}^{-} / \mathrm{gI}^{-} / \mathrm{RR}^{-}$. The virus titer at each time points and plaque size were averaged by triple samples.
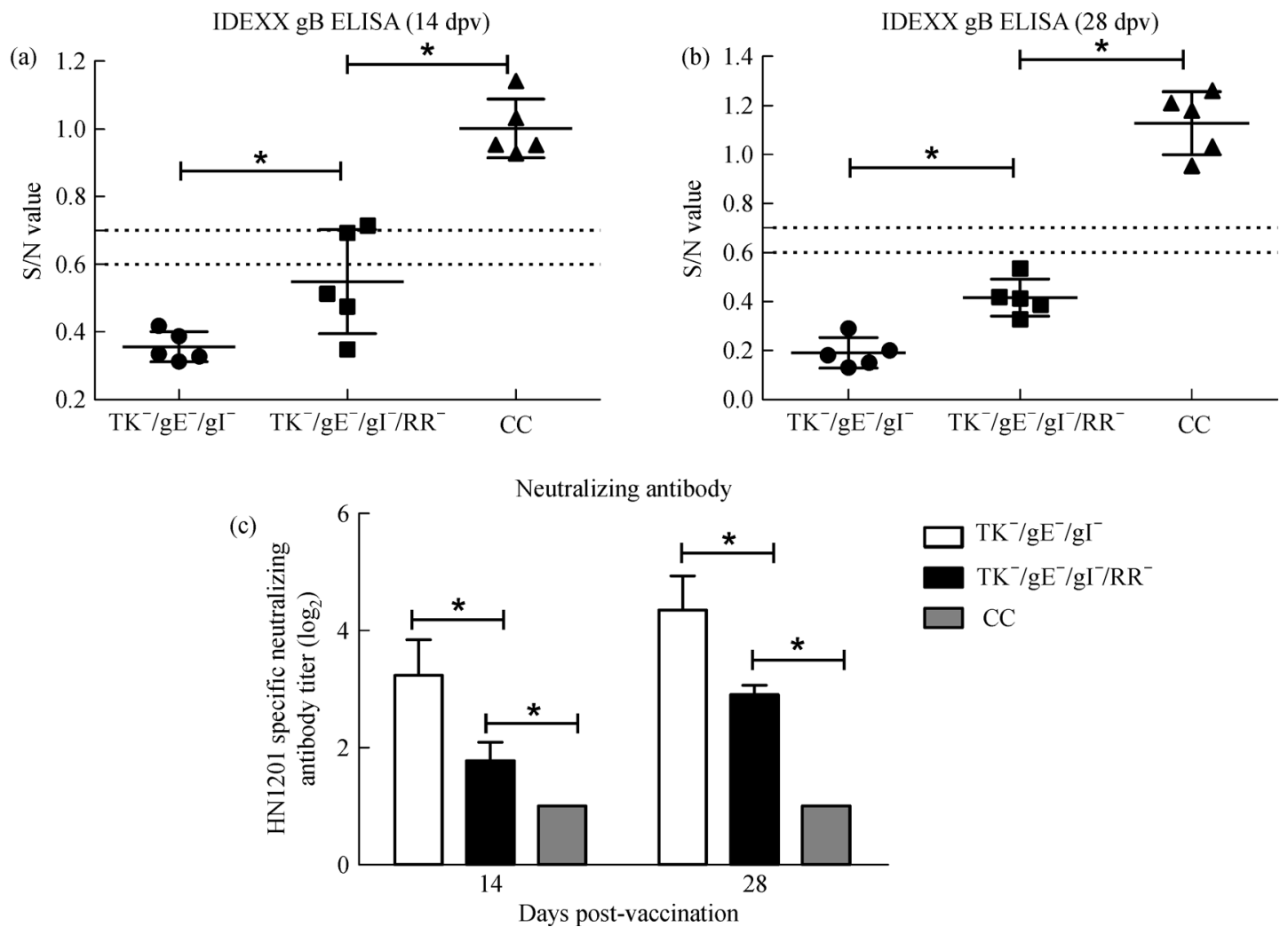

Fig. 2 PRV gB ELISA Ab responses (a, b) and PRV-specific virus neutralizing antibodies (c). The cutoff S/N values for the gB ELISA are: negative as greater than 0.7 , positive as less than or equal to 0.6 , and suspect as greater than 0.6 and less than or equal to 0.7 . $*, P<0.05$. 

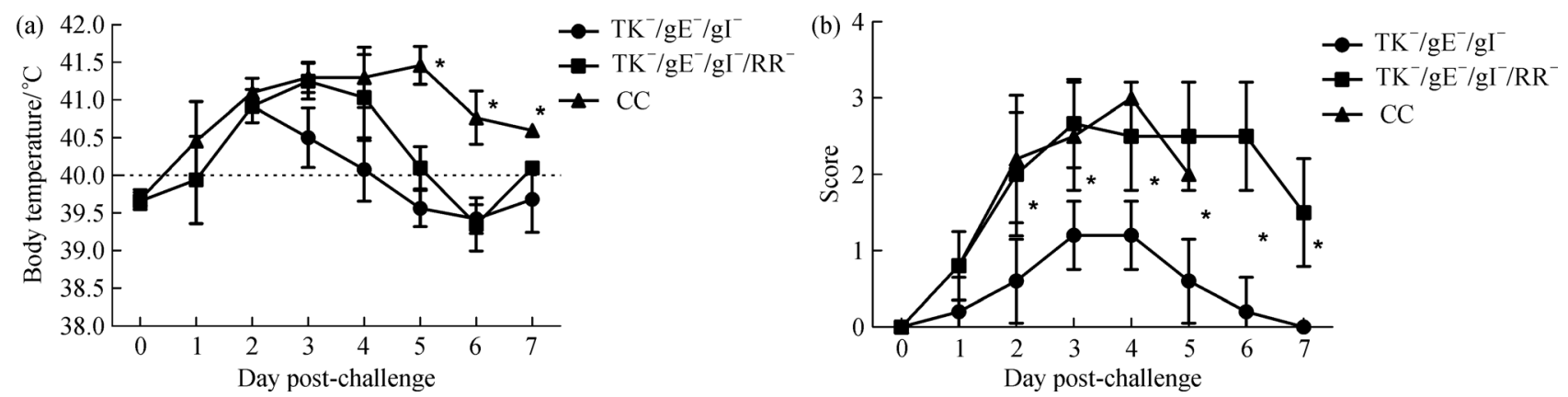

Fig. 3 Body temperature (a) and clinical symptom scores (b) of pigs after viral challenge. *, $P<0.05$.

bodyweight, with daily bodyweight loss in the DMEM group significantly higher than that in the $\mathrm{vPRV} \mathrm{TK}^{-} / \mathrm{gE}^{-} /$ $\mathrm{gI}^{-} / \mathrm{RR}^{-}$group. In contrast, pigs in the $\mathrm{vPRV} \mathrm{TK}^{-} / \mathrm{gE}^{-} / \mathrm{gI}^{-}$ group had normal daily bodyweight gain.

\subsection{Histopathology and immunohistochemistry staining}

At necropsy, all pigs in the $\mathrm{vPRV} \mathrm{TK}^{-} / \mathrm{gE}^{-} / \mathrm{gI}^{-} / \mathrm{RR}^{-}$group and DMEM groups had hemorrhage and necrosis in tonsil samples, severe pulmonary consolidation and necrosis in lung samples, and encephalic hemorrhage in brain samples. Histopathological examination confirmed hemorrhages and necrosis in tonsil and lung samples in the vPRV $\mathrm{TK}^{-} / \mathrm{gE}^{-} / \mathrm{gI}^{-} / \mathrm{RR}^{-}$and DMEM groups (Fig. 5). Neuronal degeneration and necrosis in brain and cerebellum were also observed in these two groups of pigs. Immunochemistry results showed positive staining in tonsil, lung, brain, and cerebellum samples (Fig. 5). There were no visible gross pathological and histopathological changes or positive staining of IHC for pigs in the $\mathrm{vPRV} \mathrm{TK}^{-} / \mathrm{gE}^{-} /$ $\mathrm{gI}^{-}$group.

\section{Discussion}

Gene-deleted PRV live vaccines, combined with the use of an ELISA kit which differentiated vaccinated pigs from infected ones have been widely used to eradicate diseases. A good example is PRV Barth K-61 vaccine with $g E / g I /$ US9 gene-deletion that renders the strain incapable of anterograde spread $^{[10]}$. Besides natural deletions of genes by extensively passaging field isolates in cell cultures, attenuation of PRV is also mediated by deletion of single or multiple virulence-determined genes such as $T K, g E$ and $g I$ by using BAC technology ${ }^{[4]}$.

In PRV, ribonucleotide reductase catalyzes the reduction of ribonucleotides into deoxyribonucleotides, the substrates for DNA synthesis ${ }^{[8]}$. Previous studies showed that Herpes simplex virus (HSV) strains with deletion of $R R 1$ or $R R 2$ could replicate well in cell culture but were severely attenuated in mice ${ }^{[11]}$. One study also showed that $R R 1$-deficient PRV was avirulent for pigs but induce partial protective immunity ${ }^{[12]}$. Therefore, in this study, we completely removed $R R 1$ and $R R 2$ genes to evaluate deficient PRV efficacy as a vaccine candidate.

The rescued $v \mathrm{PRV} \mathrm{TK}^{-} / \mathrm{gE}^{-} / \mathrm{gI}^{-} / \mathrm{RR}^{-}$showed similar growth kinetics and plaque size as parental virus which indicates $R R$ genes did not affect the proliferation of virus in dividing cells as previously reported ${ }^{[13]}$. However, vaccination did not provide protection against virulent PRV HN1201 challenge as all vaccinated pigs showed PRtypical clinical symptoms with high mortality. Compared to its parental virus, $\mathrm{vPRV}^{\mathrm{TK}} / \mathrm{gE}^{-} / \mathrm{gI}^{-} / \mathrm{RR}^{-}$vaccinated pigs developed late and low level of gB-specific antibodies and virus neutralizing antibodies after vaccination. $\mathrm{gB}$ antibodies and virus neutralizing antibodies have been widely used to evaluate the efficacy of PRV vaccines, and inability to generate early and protective level of these antibodies may be due to the poor replication of virus via intranasal inoculation.

A RR1-deficient HSV-1 mutant showed poor replication in non-dividing cells ${ }^{[14]}$. However, the virus growth in dividing cells was found to be much better given that deoxyribonucleotides for virus replication were provided by cellular nucleotide metabolism ${ }^{[13]}$. In pigs, a previous study reported RRl-deleted PRV mutants had poor replication in nasal and oropharyngeal mucosa but did induce low titers of neutralizing antibodies which indicated vaccination could elicit immune responses ${ }^{[12]}$. Therefore, low proliferation of virus after vaccination may partially explain the generation of late and low level of gB-specific antibodies which could lead to failure of vaccination. However, we cannot exclude the possibility that some other factors may have contributed to the observed lack of protection.

In de Wind's study, a $R R I$ mutant PRV provided protection against wild-type PRV challenge which differs from our results ${ }^{[12]}$. This discrepancy could be explained by different deletion of $R R$ genes (both $R R 1$ and $R R 2$ genes were deleted in our study) and different parental viruses used for the gene manipulation $\left(\mathrm{TK}^{-} / \mathrm{gE}^{-} / \mathrm{gI}^{-} \mathrm{PRV}\right.$ was used in this study). However, the mechanisms of inability of this virus to provide protective immunity still need further exploration. 

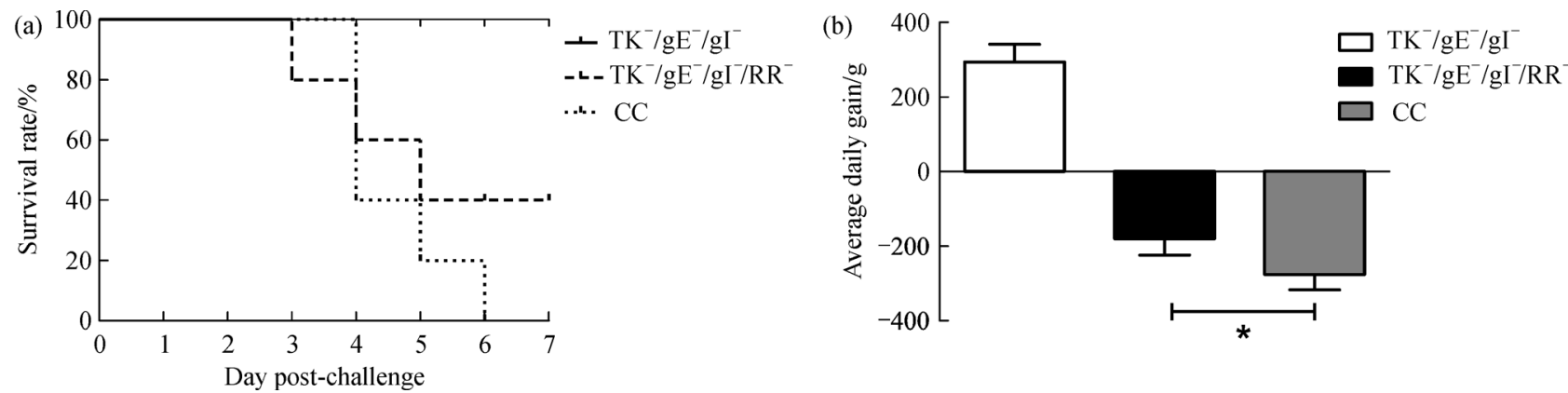

Fig. 4 Survival rate (a) and daily bodyweight gain for $4 \mathrm{dpc}$ (b) of pigs after viral challenge. ${ }^{*}, P<0.05$.
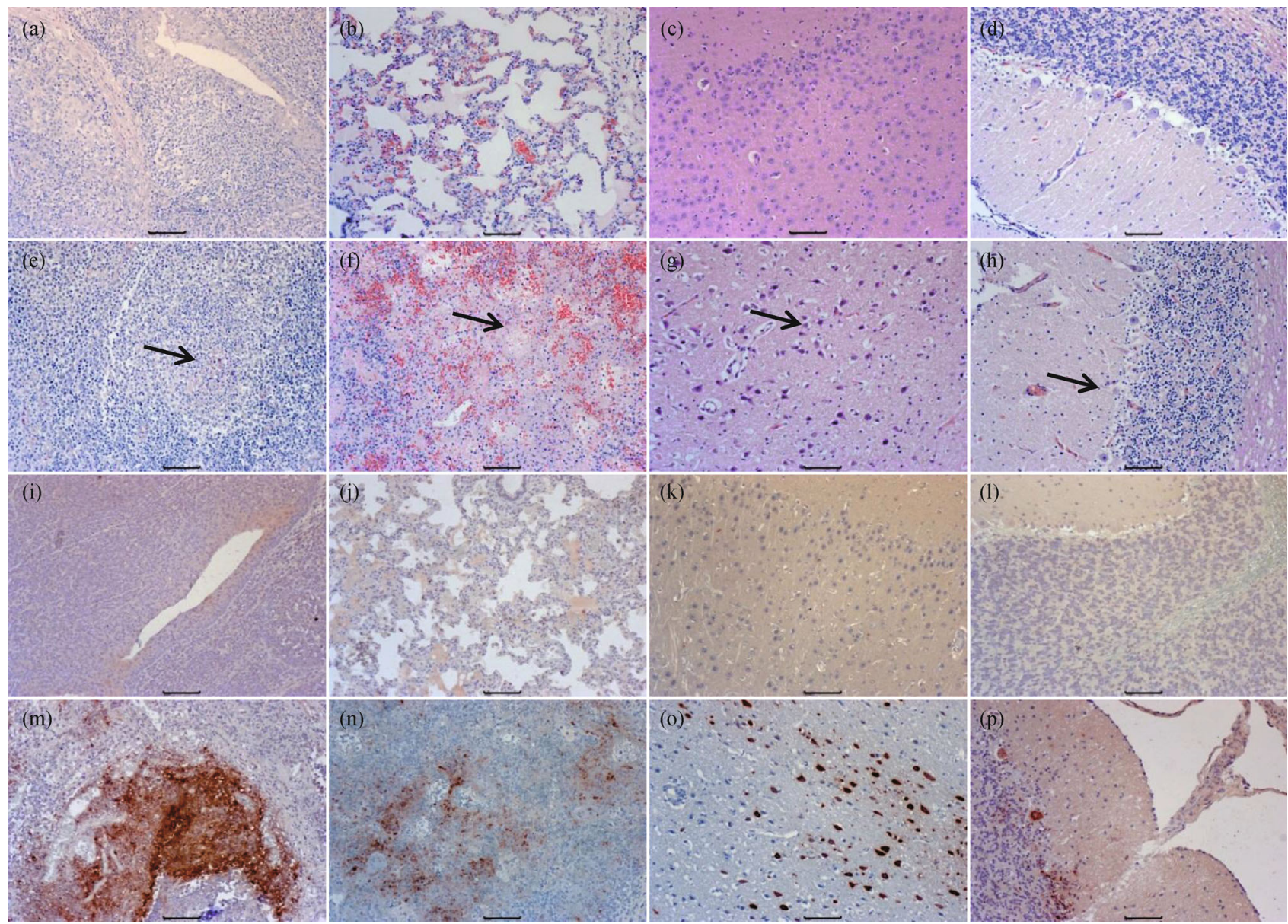

Fig. 5 Histopathological (a-h) and immunochemistry (i-p) results of $\mathrm{vPRVTK}^{-} / \mathrm{gE}^{-} / \mathrm{gI}^{-}(\mathrm{a}-\mathrm{d}, \mathrm{i}-\mathrm{l})$ and $\mathrm{vPRVTK}^{-} / \mathrm{gE}^{-} / \mathrm{gI}^{-} / \mathrm{RR}^{-}$ $(\mathrm{e}-\mathrm{h}, \mathrm{m}-\mathrm{p})$. (a-d) Tonsil, lung, brain, and cerebellum samples from the pigs in vPRVTK ${ }^{-} / \mathrm{gE}^{-} / \mathrm{gI}^{-}$group. No obvious histopathological changes were observed; (e) tonsil. Tonsillar lymphoid tissue necrosis and formation of a big necrotic foci; (f) lung. Massive vascular congestion and hemorrhage; (g) brain. Nonsuppurative meningoencephalitis; (h) cerebellum. Purkinje cell degeneration and necrosis; (i-1) negative immunochemistry staining of corresponding tonsil, lung, brain, and cerebellum samples in $\mathrm{vPRVTK}^{-} / \mathrm{gE}^{-} / \mathrm{gI}^{-} \mathrm{group}^{-}$ $(\mathrm{m}-\mathrm{p})$ positive immunochemistry staining of corresponding tonsil, lung, brain, and cerebellum samples in $\mathrm{vPRVTK}^{-} / \mathrm{gE}^{-} / \mathrm{gI}^{-} / \mathrm{RR}^{-} \mathrm{group}$. Original magnification $200 \times$.

\section{Conclusions}

To conclude, we successfully constructed the $R R 1$ and $R R 2$ gene-deleted PRV strain based on vPRV TK $/ \mathrm{gE}^{-} / \mathrm{gI}$ virus and showed it was not effective as a vaccine candidate.
Acknowledgements This work was supported by grant from Major Science and Technology Program in Henan Province (131100110200), Innovation Scientists and Technicians Troop Construction Projects of Henan Province (142101510001), Talents Plan for Scientific and Technological Innovation in Henan Province (144200510002), and Science and Technology Innovation team in Henan Province (C20130005). 
Compliance with ethics guidelines Shijun Yan, He Yan, Chaolin Zhang, Tongyan Wang, Qingyuan Yang, Zhe Sun, Yan Xiao, Feifei Tan, Xiangdong $\mathrm{Li}$, and Kegong Tian declare that they have no conflict of interest or financial conflicts to disclose.

All applicable institutional and national guidelines for the care and use of animals were followed.

\section{References}

1. Mettenleiter T C. Immunobiology of pseudorabies (Aujeszky's disease). Veterinary Immunology and Immunopathology, 1996, 54 (1-4): 221-229

2. Mettenleiter T C. Pseudorabies (Aujeszky's disease) virus: state of the art. August 1993. Acta Veterinaria Hungarica, 1994, 42: 153177

3. Tian K G, Yu X L, Zhao T Z, Feng Y J, Cao Z, Wang C B, Hu Y, Chen X Z, Hu D M, Tian X S, Liu D, Zhang S, Deng X Y, Ding Y Q, Yang L, Zhang Y X, Xiao H X, Qiao M M, Wang B, Hou L L, Wang X Y, Yang X Y, Kang L P, Sun M, Jin P, Wang S J, Kitamura Y, Yan J H, Gao G F. Emergence of fatal PRRSV variants: unparalleled outbreaks of atypical PRRS in China and molecular dissection of the unique hallmark. PLOS ONE, 2007, 2(6): e526

4. Zhang C L, Guo L H, Jia X R, Wang T Y, Wang J, Sun Z, Wang L L, Li X D, Tan F F, Tian K G. Construction of a triple gene-deleted Chinese pseudorabies virus variant and its efficacy study as a vaccine candidate on suckling piglets. Vaccine, 2015, 33(21): 2432 2437

5. Gu Z, Dong J, Wang J, Hou C, Sun H, Yang W, Bai J, Jiang P. A novel inactivated $\mathrm{gE} / \mathrm{gI}$ deleted pseudorabies virus (PRV) vaccine completely protects pigs from an emerged variant PRV challenge. Virus Research, 2015, 195: 57-63

6. Wang C H, Yuan J, Qin H Y, Luo Y, Cong X, Li Y, Chen J, Li S, Sun Y, Qiu H J. A novel gE-deleted pseudorabies virus (PRV) provides rapid and complete protection from lethal challenge with the PRV variant emerging in Bartha-K61-vaccinated swine popula- tion in China. Vaccine, 2014, 32(27): 3379-3385

7. Pomeranz L E, Reynolds A E, Hengartner C J. Molecular biology of pseudorabies virus: impact on neurovirology and veterinary medicine. Microbiology and Molecular Biology Reviews, 2005, 69(3): 462-500

8. Kaliman A V, Boldogkoi Z, Fodor I. Large and small subunits of the Aujeszky's disease virus ribonucleotide reductase: nucleotide sequence and putative structure. Biochimica et Biophysica Acta, 1994, 1219(1): 151-156

9. Wang T Y, Xiao Y, Yang Q Y, Wang Y Z, Sun Z, Zhang C L, Yan S J, Wang J, Guo L H, Yan H, Gao Z Y, Wang L L, Li XD, Tan F F, Tian KG. Construction of a gE-deleted pseudorabies virus and its efficacy to the new-emerging variant PRV challenge in the form of killed vaccine. BioMed Research International, 2015, 27: 1-10

10. Curanovic D, Lyman M G, Bou-Abboud C, Card J P, Enquist L W. Repair of the UL21 locus in pseudorabies virus Bartha enhances the kinetics of retrograde, transneuronal infection in vitro and in vivo. Journal of Virology, 2009, 83(3): 1173-1183

11. de Wind N, Peeters B P H, Zijderveld A, Gielkens A L, Berns A J M, Kimman T S. Mutagenesis and characterization of a 41-kilobasepair region of the pseudorabies virus genome: transcription map, search for virulence genes, and comparison with homologs of herpes simplex virus type 1. Virology, 1994, 200(2): 784-790

12. de Wind N, Berns A, Gielkens A, Kimman T. Ribonucleotide reductase-deficient mutants of pseudorabies virus are avirulent for pigs and induce partial protective immunity. Journal of General Virology, 1993, 74(3): 351-359

13. Goldstein D J, Weller S K. Herpes simplex virus type 1-induced ribonucleotide reductase activity is dispensable for virus growth and DNA synthesis: isolation and characterization of an ICP6 lacZ insertion mutant. Journal of Virology, 1988, 62: 196-205

14. Preston V G, Darling A J, McDougall I M. The herpes simplex virus type 1 temperature-sensitive mutant ts 1222 has a single base pair deletion in the small subunit of ribonucleotide reductase. Virology, 1988, 167: 458-467 